\title{
Planetary-Geometric Constraints on Isopycnal Slope in the Southern Ocean
}

\author{
DANIEL C. JONES \\ British Antarctic Survey, Natural Environment Research Council, Cambridge, United Kingdom, and School of Earth \\ and Atmospheric Sciences, Georgia Institute of Technology, Atlanta, Georgia
}

TAKAMITSU ITO

School of Earth and Atmospheric Sciences, Georgia Institute of Technology, Atlanta, Georgia

THOMAS BIRNER

Department of Atmospheric Science, Colorado State University, Fort Collins, Colorado

ANDREAS KLOCKER

Institute for Marine and Antarctic Studies, University of Tasmania, Hobart, Tasmania, Australia

DAVID MUNDAY

British Antarctic Survey, Natural Environment Research Council, Cambridge, and Department of Physics, University of Oxford, Oxford, United Kingdom

(Manuscript received 16 February 2015, in final form 12 September 2015)

\begin{abstract}
On planetary scales, surface wind stress and differential buoyancy forcing act together to produce isopycnal surfaces that are relatively flat in the tropics/subtropics and steep near the poles, where they tend to outcrop. Tilted isopycnals in a rapidly rotating fluid are subject to baroclinic instability. The turbulent, mesoscale eddies generated by this instability have a tendency to homogenize potential vorticity (PV) along density surfaces. In the Southern Ocean (SO), the tilt of isopycnals is largely maintained by competition between the steepening effect of surface forcing and the flattening effect of turbulent, spatially inhomogeneous eddy fluxes of PV. Here quasigeostrophic theory is used to investigate the influence of a planetary-geometric constraint on the equilibrium slope of tilted density/buoyancy surfaces in the SO. If the meridional gradients of relative vorticity and PV are small relative to $\beta$, then quasigeostrophic theory predicts $d s / d z=\beta / f_{0}=\cot \left(\phi_{0}\right) / a$, or equivalently $r \equiv\left|\partial_{z} s /\left(\beta / f_{0}\right)\right|=1$, where $f$ is the Coriolis parameter, $\beta$ is the meridional gradient of $f, s$ is the isopycnal slope, $\phi_{0}$ is a reference latitude, $a$ is the planetary radius, and $r$ is the depth-averaged criticality parameter. It is found that the strict $r=1$ condition holds over specific averaging volumes in a large-scale climatology. A weaker $r=O(1)$ condition for depth-averaged quantities is generally satisfied away from large bathymetric features. The $r=O(1)$ constraint is employed to derive a depth scale to characterize large-scale interior stratification, and an idealized sector model is used to test the sensitivity of this relationship to surface wind forcing. Finally, the possible implications for eddy flux parameterization and for the sensitivity of SO circulation/stratification to changes in forcing are discussed.
\end{abstract}

\section{Introduction}

The Southern Ocean is a unique and dynamic component of Earth's climate system. As an important site

Corresponding author address: Dan Jones, British Antarctic Survey, High Cross, Madingley Road, Cambridge CB3 0ET, United Kingdom.

E-mail: dannes@bas.ac.uk of mode-water, intermediate-water, and deep-water formation, the Southern Ocean hosts a dominant transport pathway between the atmosphere and the interior ocean (Russell et al. 2006). This pathway is set in part by steeply tilted isopycnal surfaces that outcrop at high southern latitudes. Through this window, atmospheric carbon is exchanged with the interior ocean, potentially slowing the buildup of anthropogenic carbon dioxide in the atmosphere while altering the 
biogeochemistry of the global ocean (Takahashi et al. 2009). Since mean stratification (and the closely related slope of Southern Ocean isopycnals) is likely to impact abyssal circulation and deep-water transport, the largescale density structure of the Southern Ocean could be considered an important "state variable" of both global ocean circulation and the larger climate system.

The steep tilt of isopycnals in the Southern Ocean is established and maintained at least in part by (i) convergences and divergences of wind-driven Ekman flow in the surface ocean and (ii) the planetary-scale meridional buoyancy gradient. The zonal mean forcing pattern varies with latitude in such a way that isopycnals are tilted up toward the Antarctic continent (i.e., they outcrop in the south and plunge into the interior toward the subtropics). They join smoothly with the subtropical density structure, which is characterized by relatively flat isopycnals. Climatological mean potential density is shown in Fig. 1.

Tilted isopycnals like those found in the Southern Ocean are susceptible to baroclinic instability. Mesoscale eddies generated by baroclinic instability have a tendency to homogenize potential vorticity (PV) along isopycnals via downgradient thickness fluxes, flattening constant density surfaces in the process (Marshall and Speer 2012). The mean slope of interior Southern Ocean isopycnals is thought to be set by a balance between the steepening effect of wind stress and buoyancy fluxes and the flattening effect of energetic, mesoscale eddies (Karsten and Marshall 2002). This balance affects many facets of global ocean circulation across different time scales, including the sensitivity of the overturning circulation to changes in Southern Hemispheric wind stress and the strength of the Southern Ocean carbon sink (Lovenduski and Ito 2009; Abernathey et al. 2011; Munday et al. 2014).

In this work, we investigate the influence of a planetarygeometric constraint on the equilibrium slope of tilted buoyancy surfaces in the Southern Ocean. Using zonal mean theory with quasigeostrophic dynamics, we derive a relationship between the large-scale, depthaveraged vertical gradient of isopycnal slope and the ratio $\beta / f_{0}$ [where $\beta=d f / d y, f=2 \Omega \sin (\phi)$ is the Coriolis parameter, $\Omega$ is the planetary rotation rate in radians per second, $\phi$ is latitude, and $f_{0}$ is $f$ evaluated about a reference latitude $\left.\phi_{0}\right]$. This relationship suggests that if the depth-averaged quasigeostrophic potential vorticity (QGPV) gradient is small relative to $\beta$, then the vertical gradient of isopycnal slope (i.e., the rate of change of slope with depth) is set by $\beta / f_{0}$. We find that in climatological Southern Ocean reanalysis fields, the vertical gradient of isopycnal slope is of order $\beta / f_{0}$, and we use this condition to derive a scale for the vertical gradient
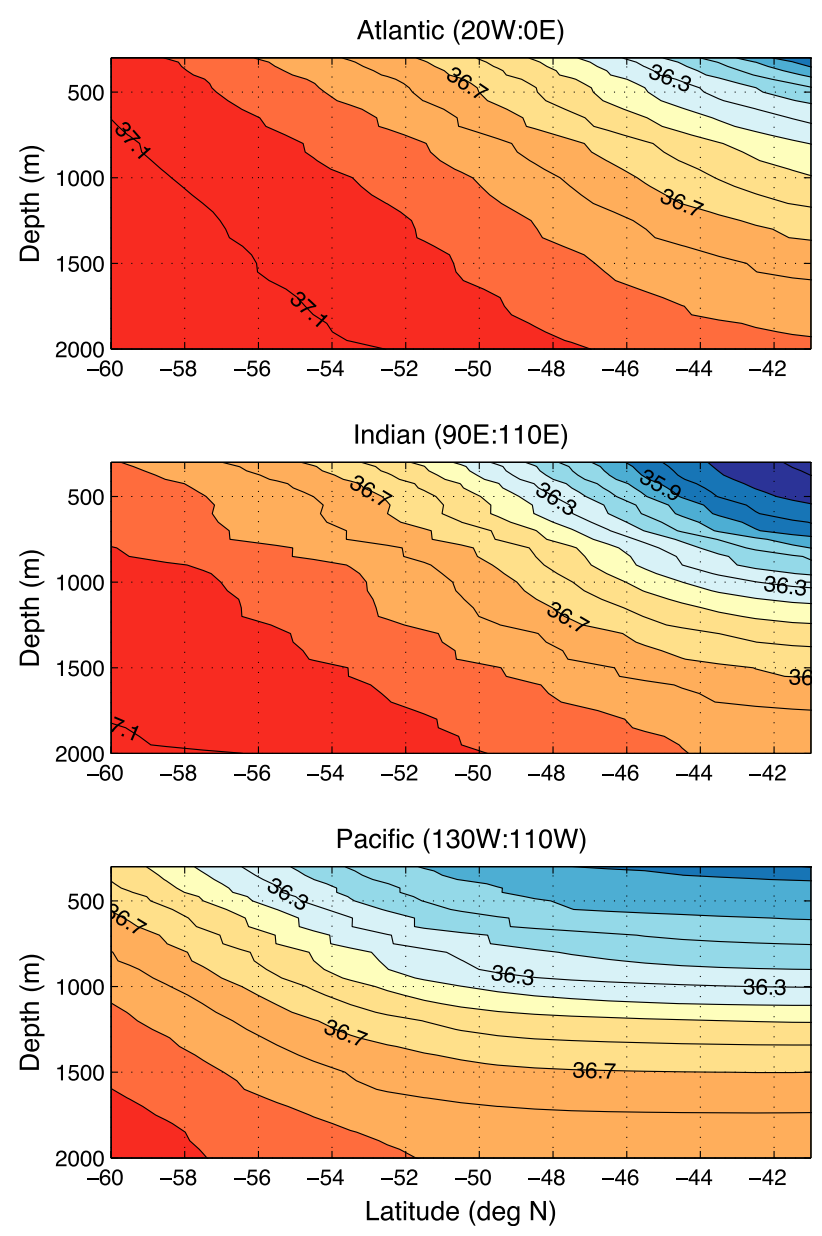

FIG. 1. Zonal mean potential density referenced to $2000 \mathrm{dbar}\left(\sigma_{2}\right.$; $\mathrm{kg} \mathrm{m}^{-3}$ ) for the indicated longitude ranges and from $40^{\circ}$ to $60^{\circ} \mathrm{S}$. Contours are spaced every $0.1 \mathrm{~kg} \mathrm{~m}^{-3}$. Data are from World Ocean Atlas 2013.

of isopycnal slope. The Pacific Ocean features the weakest vertical gradients of isopycnal slope relative to the Indian and Atlantic sectors of the Southern Ocean. Finally, we use an idealized sector model to examine the sensitivity of stratification to changes in surface wind forcing and eddy activity. The vertical gradient of isopycnal slope is relatively insensitive to surface wind stress in the presence of permitted mesoscale eddies.

\section{Theoretical justification}

\section{a. Basic assumptions and definitions}

We consider an idealized ocean with no longitudinally oriented barriers such that zonal mean theory is generally applicable (Marshall and Radko 2003). Zonal mean buoyancy [simply written as $b=b(y, z, t)$ in this section in order to keep the notation from getting cluttered] can be decomposed into the average buoyancy, $\tilde{b}(z)$, and the 
departures therefrom, $b^{\prime}(y, z, t)$. That is, we can write $b(y, z, t)=\tilde{b}(z)+b^{\prime}(y, z, t)$. We define vertical stratification as $N^{2}(z)=\partial_{z} \tilde{b}$ (i.e., the square of the buoyancy frequency) and horizontal stratification as $M^{2}(y, z, t)=$ $\partial_{y} b^{\prime}=\partial_{y} b$. The last equation exploits the assumption that the average buoyancy $\tilde{b}(z)$ does not depend on $y$. We assume that $\left|\partial_{z} b^{\prime}\right| \ll\left|\partial_{z} \tilde{b}\right|$, such that vertical variations in $b$ are well approximated by vertical variations in the average buoyancy field $\tilde{b}=\tilde{b}(z)$. Therefore, we can relate the slope of zonal mean buoyancy surfaces $s_{b}$ to the stratification by $M^{2} / N^{2}=\partial_{y} b / \partial_{z} \tilde{b} \approx \partial_{y} b / \partial_{z} b=-s_{b}$ [the last equation follows from examining the total differential of $b$ on a buoyancy surface, i.e., $\delta b=\partial_{y} b \delta y+$ $\partial_{z} b \delta z=0$, where $s_{b}=(\delta z / \delta y)_{b}$ at constant buoyancy $\left.b\right]$.

In a continuously stratified fluid with relative vorticity $\zeta_{g}=(\nabla \times \mathbf{v}) \cdot \mathbf{k}$, where $\mathbf{v}$ is the velocity vector and $\mathbf{k}$ is the vertical unit vector, the quasigeostrophic potential vorticity is given by

$$
q=\zeta_{g}+\beta y+\partial_{z}\left(f_{0} N^{-2} b^{\prime}\right),
$$

where $\beta=\partial_{y} f$ is the meridional gradient of the Coriolis parameter [i.e., $f=2 \Omega \sin (\phi)$, where $\Omega$ is the planetary rotation rate and $\phi$ is the latitude (Vallis 2006, Eq. (5.133)]. Since we are working on a $\beta$ plane, the Coriolis parameter increases linearly as $f=f_{0}+\beta y$. Taking the meridional gradient of Eq. (1) on surfaces of constant depth, we get

$$
\partial_{y} q=\partial_{y} \zeta_{g}+\beta+\partial_{z}\left(f_{0} N^{-2} \partial_{y} b^{\prime}\right) .
$$

Since $N^{-2} \partial_{y} b^{\prime}=M^{2} / N^{2} \approx-s_{b}$, we can write Eq. (2) as

$$
\partial_{y} q=\partial_{y} \zeta_{g}+\beta-f_{0} \partial_{z} s_{b} .
$$

Averaging Eq. (3) with depth and dividing through by $\beta$, we get

$$
\frac{\overline{\partial_{y} q}}{\beta}=1+\frac{\overline{\partial_{y} \zeta_{g}}}{\beta}-\frac{f_{0} \overline{\partial_{z} s_{b}}}{\beta}
$$

where the overbars denote depth averaging over a chosen vertical scale. Equation (4) is essentially a depth-averaged variant of the large-scale quasigeostrophic potential vorticity gradient as presented in Bretherton (1966). Here we use quasigeostrophic theory on surfaces of constant depth (as opposed to the more general version on surfaces of constant density or neutral surfaces) because our scaling is especially clean in the $z$-level coordinate system.

\section{b. The large-scale vertical gradient of buoyancy surfaces}

Here we assume that the large-scale, depth-averaged meridional gradients of potential vorticity and relative vorticity are small relative to $\beta$ in the interior Southern Ocean across the latitudes of the Antarctic Circumpolar Current (ACC). We will revisit these assumptions in a later section. In this assumed limit, the dominant scaling for Eq. (4) is $\overline{\partial_{z} s_{b}}=\beta / f_{0}$. The ratio of the mean slope gradient (i.e., $\overline{\partial_{z} s_{b}}$ ) and planetary parameters (i.e., $\beta / f_{0}$ ) can be written as

$$
r \equiv\left|\frac{\overline{\partial_{z} s_{b}}}{\beta / f_{0}}\right|,
$$

where $r$ is the depth-averaged version of the criticality parameter based on the condition for marginal criticality in the two-layer quasigeostrophic model (Stone 1978), with $r>1$ being the criterion for baroclinic instability. ${ }^{1}$ The ratio $r$ can also be interpreted as a rough bulk metric for the vertical gradient of isopycnal slope relative to $\beta / f_{0}$. In the $r=1$ regime, the vertical gradient of isopycnal slope is constrained, remarkably, by a ratio of planetary-geometric factors:

$$
\overline{\left(\frac{\partial s}{\partial z}\right)} \approx \frac{\beta}{f_{0}}=\frac{2 \Omega a^{-1} \cos \left(\phi_{0}\right)}{2 \Omega \sin \left(\phi_{0}\right)}=a^{-1} \cot \left(\phi_{0}\right),
$$

where $a$ is the radius of the planet and $\phi_{0}$ is a reference latitude. This can also be explained by saying that the QGPV gradient vanishes due to a cancellation between the planetary vorticity gradient $\beta$ and the "thickness" gradient $f_{s} / H$. The rotation rate $\Omega$ has dropped out of the equation entirely, but the assumption of rapid rotation is still necessary since we are operating in a quasigeostrophic framework.

Values of $r<1$ indicate that the buoyancy surfaces are "undertilted" with respect to $\beta / f_{0}$. In this regime, depthaveraged potential vorticity increases with latitude (i.e., $\left.\overline{\partial_{y} q}>0\right)$ and the potential vorticity gradient is dominated by rotation effects. The case $r=0$ corresponds to uniformly tilted buoyancy surfaces (i.e., zero vertical gradient of isopycnal slope). Values of $r>1$ indicate that buoyancy surfaces are "overtilted" with respect to $\beta / f_{0}$. In this regime, depth-averaged potential vorticity decreases with latitude (i.e., $\overline{\partial_{y} q}<0$ ) and the potential vorticity gradient is dominated by stratification effects. Values of $r=1$ indicate that in a depth-averaged sense, rotation and stratification are equally important, which in an energetically favored state leads to length scales $O\left(L_{D}\right)$, where $L_{D}$ is the baroclinic deformation radius.

\footnotetext{
${ }^{1}$ See the discussion in Jansen and Ferrari (2012) and references therein on marginal criticality scaling and its applicability to a continuously stratified model.
} 
We can express the depth-averaged change in slope with depth as

$$
\overline{\left(\frac{\partial s}{\partial z}\right)}=\frac{\int_{0}^{H_{d}} \partial_{z} s d z}{H_{d}}=\frac{s\left(H_{d}\right)-s(0)}{H_{d}} \approx \frac{\beta}{f_{0}}=a^{-1} \cot \left(\phi_{0}\right),
$$

where $H_{d}$ is the depth-averaging scale. We can also write $s\left(H_{d}\right)=s(0)+H_{d} a^{-1} \cot \left(\phi_{0}\right)$, where $H_{d}$ must be large enough such that the $r=1$ scaling holds. This deceptively simple linear equation must be interpreted with care. Equation (7) does not predict a linear change in slope with depth everywhere in the domain, but it does predict that the depth-averaged vertical gradient of isopycnal slope is constrained by a planetary-geometric parameter, which is mathematically consistent with an infinite number of different density structures.

Furthermore, the planetary-geometric constraint only influences the rate of change of the slope of buoyancy/ density surfaces with depth; it does not fix the actual value of slope, which is strongly influenced by air-sea interactions and mixing. The term $s\left(H_{d}\right)$ in Eq. (7) is not necessarily zero, since we have chosen our domain to lie above the bathymetry and sea floor. Similarly, $s(0)$ is only the slope of buoyancy surfaces at the top of the domain, which is taken to be well below the mixed layer; it is not intended to represent the slope at the surface of the ocean.

In the following sections, we estimate $r$ and estimate a depth scale over which slope changes significantly using both observationally derived datasets and an idealized interhemispheric sector model.

\section{Data and methods}

\section{a. Climatology}

We employ objectively analyzed climatological mean temperature and salinity fields derived from nearly six decades of in situ profile data (Locarnini et al. 2010; Zweng et al. 2013). The fields are on a $1^{\circ} \times 1^{\circ}$ global grid with 102 vertical levels from the surface down to $5500 \mathrm{~m}$. Each climatological field is a nearly six-decadal mean taken over the period 1955-2012. Therefore, the fields only represent the long-term, large-scale average structure of temperature and salinity, and some interpolation has been used to fill in missing values. These fields are part of the World Ocean Atlas 2013 (WOA13) suite, which for this work have been retrieved from the National Oceanographic Data Center (NODC; http://www.nodc.noaa. gov/OC5/woa13/). Density is calculated using the modified United Nations Educational, Scientific and Cultural Organization (UNESCO) polynomial of Jackett and
Mcdougall (1995, hereinafter JMD95). Since WOA13 has relatively low spatial resolution and does not include velocity fields, we also use 6-yr-averaged (2005-10) zonal and meridional velocity fields from the Southern Ocean State Estimate (SOSE), which are on a $1 / 6^{\circ}$ horizontal grid with 42 vertical levels (Mazloff et al. 2010).

\section{b. Sector model setup}

The sector model is an idealized configuration of the MIT general circulation model (MITgcm) designed to allow a large number of numerical experiments to be run to equilibrium at a range of resolutions and wind forcings (Marshall et al. 1997a,b). Full details of the configuration are given in Marshall et al. (1997a,b), Munday et al. (2013), Hogg and Munday (2014), and Munday et al. (2014), although a brief exposition follows.

The sector model domain stretches from $60^{\circ} \mathrm{S}$ to $60^{\circ} \mathrm{N}$ and is $20^{\circ}$ in longitude wide. A "circumpolar" channel extends over the southernmost $20^{\circ}$ of latitude and an extra 1 grid point, or $1^{\circ}$ in longitude, whichever is greater, forms the model's "Southern Ocean." Within this extra section, the depth is $2500 \mathrm{~m}$, but is otherwise $5000 \mathrm{~m}$ throughout the rest of the domain. The step is sufficiently high so as to block all $f / H$ contours. When the model grid spacing is fine enough to permit or resolve the mesoscale eddy field, this allows surface wind stress to be balanced by bottom form stress in the expected momentum balance for the Southern Ocean (e.g., Munk and Palmén 1951). In this balance, interfacial eddy form stresses transmit momentum vertically through the water column.

The sector model is driven at the surface by an idealized profile of wind stress that places the peak wind stress within the circumpolar channel [see Fig. 2 of Munday et al. (2013)]. Surface forcing of temperature and salinity is carried out using restoring toward idealized profiles based on observations of the Atlantic. The restoring time scale for temperature is 10 days, and the restoring time scale for salinity is 30 days. The structure of the surface forcing does not vary with model grid spacing and is designed so that the surface density at the northern boundary is intermediate between that at the southern boundary and the northern edge of the circumpolar channel. This ensures that the model analog of North Atlantic Deep Water sinks to middepth and upwells within the confines of the circumpolar channel.

At a grid spacing of $2^{\circ}$, the sector model uses the Gent and McWilliams (1990) parameterization of the mesoscale eddy field with a constant diffusivity coefficient of $1000 \mathrm{~m}^{2} \mathrm{~s}^{-1}$. At finer grid spacings, the coefficient is greatly reduced, such that the permitted/resolved mesoscale eddy field is undamped by the parameterization [see Munday et al. (2013) for details].

At each model grid spacing, a total of 12 different wind stress values were used, ranging from a peak value 

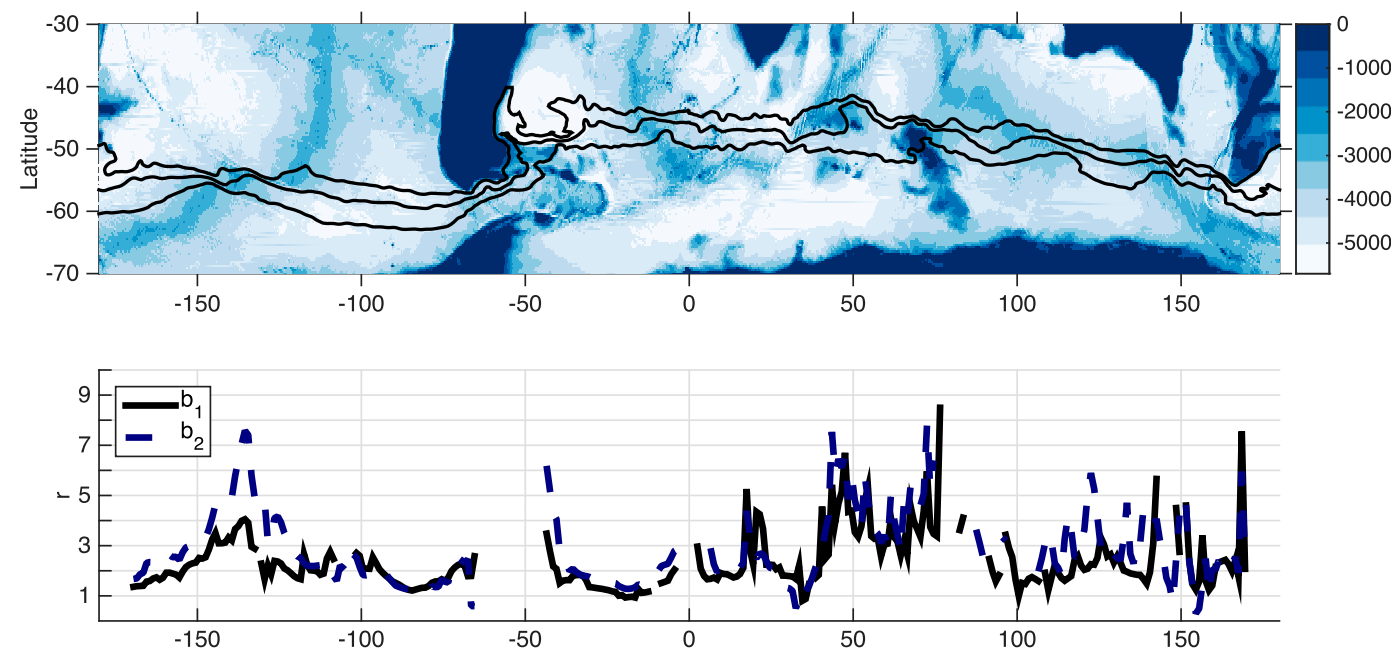

FIG. 2. (top) Southern Ocean bathymetry with three fronts of the Antarctic Circumpolar Current, namely the Polar Front (PF, southernmost), Subantarctic Front (SAF, middle), and the northern extension of the Subantarctic Front (SAF-N, northernmost). Depth scale is shown in meters. (bottom) Value of $r=\left|\overline{\partial_{z} s} /\left(\beta / f_{0}\right)\right|$ calculated across the fronts of the ACC using the slope of potential buoyancy $b_{\sigma}$ using $\sigma_{1}$ (solid black line) and $\sigma_{2}$ (dashed blue line) to define density surfaces in $10^{\circ}$-wide zonal mean bins. Values where $r>10$ are not shown (e.g., around the Kerguelen Plateau).

of $0.0 \mathrm{~N} \mathrm{~m}^{-2}$ to a peak value of $1.0 \mathrm{~N} \mathrm{~m}^{-2}$ with a control value of $0.2 \mathrm{~N} \mathrm{~m}^{-2}$. The $2^{\circ}$ model was run for 30000 years in total and is at equilibrium in under half of this (roughly 10000 years). The $1 / 6^{\circ}$ model was run for 400 years, which is long enough for the isopycnal slopes and "Drake Passage" transport to be reasonably equilibrated. The control experiment, and both extreme wind perturbations, were run for another 400 years and the (small) change in 10-yr mean circumpolar transport was well within the variability.

The $1 / 6^{\circ}$ model has a sufficiently fine grid so as to permit a vigorous mesoscale eddy field. At the northern edge of the circumpolar channel, the first baroclinic Rossby radius is around $40-60 \mathrm{~km}$, roughly $4-5$ grid boxes. The deformation radius is much smaller near the southern boundary and the flow commensurately less well resolved. The eddy kinetic energy (EKE) is comparable to that found in state-of-the-art ocean simulations, such as the coupled climate models in Delworth et al. (2012) or the latest iteration of the SOSE (Mazloff et al. 2010). Details of the spatial distribution and sensitivity to wind stress changes of the EKE can be found in Munday et al. (2013).

\section{Observational and numerical tests of constraint}

\section{a. Vertical gradient of isopycnal slope}

Along several large sections of the ACC, far from large bathymetric features (e.g., in the eastern Pacific sector), the depth-averaged slope gradient (i.e., $\overline{\partial s / \partial z}$ ) is constrained by the planetary-geometric parameter $\beta / f_{0}=$ $a^{-1} \cot (\phi)$ (see Fig. 2). To illustrate this, we calculate $\partial s / \partial z$ using the slope of several potential buoyancy surfaces that cut through the interior ocean across the ACC. Density is calculated using the modified UNESCO polynomial of JMD95, and pressure is taken to be hydrostatic. Potential buoyancy is calculated as $b_{\sigma_{n}}=-g \sigma_{n} / 1000$, with potential density $\sigma_{n}$ as either $\sigma_{1}=\rho(T, S, p=1000 \mathrm{dbar})-\rho_{0}$ or $\sigma_{2}=\rho(T, S, p=2000 \mathrm{dbar})-\rho_{0}$, where $\rho_{0}=$ $1000 \mathrm{~kg} \mathrm{~m}^{-3}$ ). We consider the buoyancy surfaces in $20^{\circ}$ wide-centered zonal means (i.e., $10^{\circ}$ to either side of a selected longitude), bounded in latitude by the Polar Front (PF) and the northern extension of the Subantarctic Front (SAF-N), and bounded in depth well below the mixed layer $(500 \mathrm{~m})$ and above the bathymetry (down to $4800 \mathrm{~m}$ or just above the local bathymetry). The slope of each density surface is estimated by linear regression in order to capture the large-scale change in slope by filtering out small-scale variations. The rate of change of slope with $b$ is estimated by linear regression of slopes and buoyancy levels. Finally, the large-scale rate of change of slope with depth (i.e., $\partial b / \partial z$ ) is used to convert $\partial s / \partial b$ to $\partial s / \partial z$. In the following text, $r_{1}$ refers to $r$ evaluated using potential density surfaces $\sigma_{1}$, and $r_{2}$ refers to $r$ evaluated using $\sigma_{2}$.

The mean value of $r_{1}$ across all longitudes is 2.4 , the median value is 2.0 , and the standard deviation is 1.2 . The values of $r_{1}$ and $r_{2}$ are broadly similar across all longitudes, with some exceptions where the ACC passes over the east Pacific Rise. The mean value of $r_{2}$ across all longitudes is 2.8 , the median value is 2.4 , and the standard deviation is 1.4. The value of $r$ is somewhat 

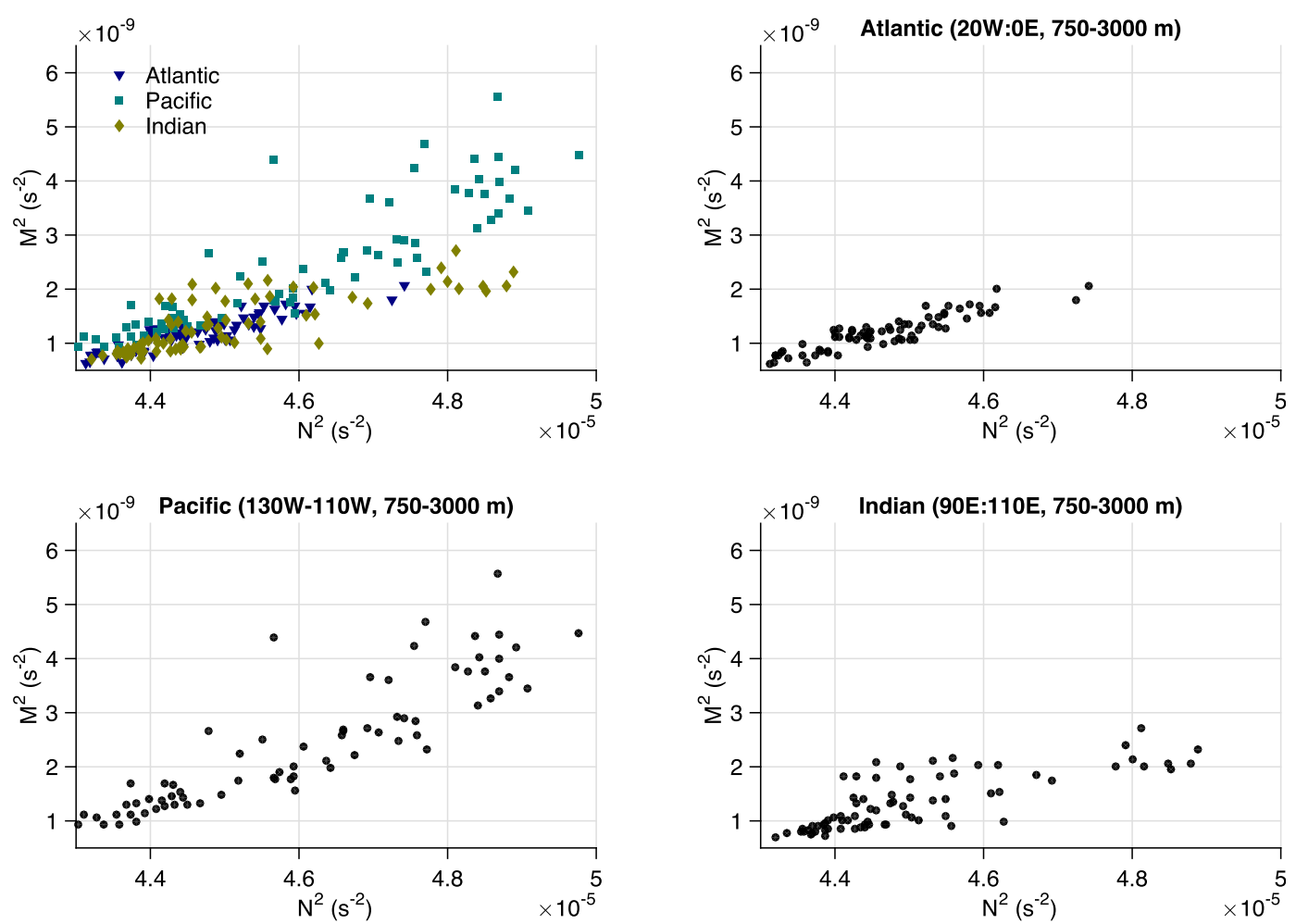

FIG. 3. Horizontal stratification $M^{2}$ vs vertical stratification $N^{2}$ in the Atlantic $\left(20^{\circ} \mathrm{W}-0^{\circ}\right)$, Indian $\left(90^{\circ}-110^{\circ} \mathrm{E}\right)$, and Pacific $\left(130^{\circ}-110^{\circ} \mathrm{W}\right)$ sectors of the Southern Ocean. Each point represents a sample from a chosen depth and latitude from a zonal mean buoyancy field.

sensitive (within roughly a factor of 1.5-2) to the choice of upper boundary (500-800 m), but it remains order 1 as predicted by quasigeostrophic scaling (which we do not expect to hold exactly). The ratio $r$ tends to be larger near bathymetric features due to the stabilizing topographic $\beta$ effect. That is, the effective topographic $\beta$ is larger near a topographic slope, which stabilizes the flow and allows for steeper isopycnals. The inclusion of the topographic $\beta$ effect might lower the value of $r$, but detailed analysis of this effect is beyond the scope of this paper.

We also calculated $r_{1}$ and $r_{2}$ using the Gibbs Seawater (GSW) Oceanographic Toolbox. The GSW mean values remained within $10 \%$ of the values obtained using JMD95, and the standard deviations changed by less than $1 \%$. GSW and JMD95 produced very similar profiles of $r$ with longitude (not shown).

\section{b. Vertical and horizontal stratification}

Next, we examine coupling between the vertical and horizontal stratification, which is an alternative way to discuss the slope of buoyancy and density surfaces. We can use Eq. (6) to construct a simple, finite-difference relationship between the vertical rate of change of slope and $\beta / f_{0}$ :

$$
\frac{\Delta s}{\Delta z}=-\frac{1}{H} \frac{M^{2}}{N^{2}}=\frac{\beta}{f_{0}},
$$

where $H$ is the vertical scale over which the slope changes appreciably. Equation (8) can be arranged as $M^{2}=\left(\beta H\left|f_{0}\right|^{-1}\right) N^{2}+\varepsilon$, where $\varepsilon$ is an error term. Here, we examine the extent to which the vertical and horizontal stratification in various sections of the Southern Ocean satisfy this linear relationship in a long-term, large-scale sense. We calculate $N^{2}$ using a simple finite-difference equation (i.e., $N^{2}=\Delta b / \Delta z$ ), which is calculated at each latitude, longitude, and depth and is then zonally averaged in a $20^{\circ}$-wide moving window. To keep the averaging scale roughly similar for $N^{2}$ and $M^{2}$, we calculate $M^{2}$ by examining the linear regression coefficient between $b$ and latitude (expressed in meters) at each depth and longitude.

In Fig. 3, we plot $M^{2}$ versus $N^{2}$ for three different sections of the Southern Ocean between $50^{\circ}-60^{\circ} \mathrm{S}$ latitude and 750-3000-m depth. Although there is some scatter, there is a linear component in the relationship between $M^{2}$ and $N^{2}$ in each basin (i.e., $p<0.01$ for a linear model). The coefficient of determination $R^{2}$ is 0.8 for the Atlantic basin, 0.8 for the Pacific basin, and 0.6 

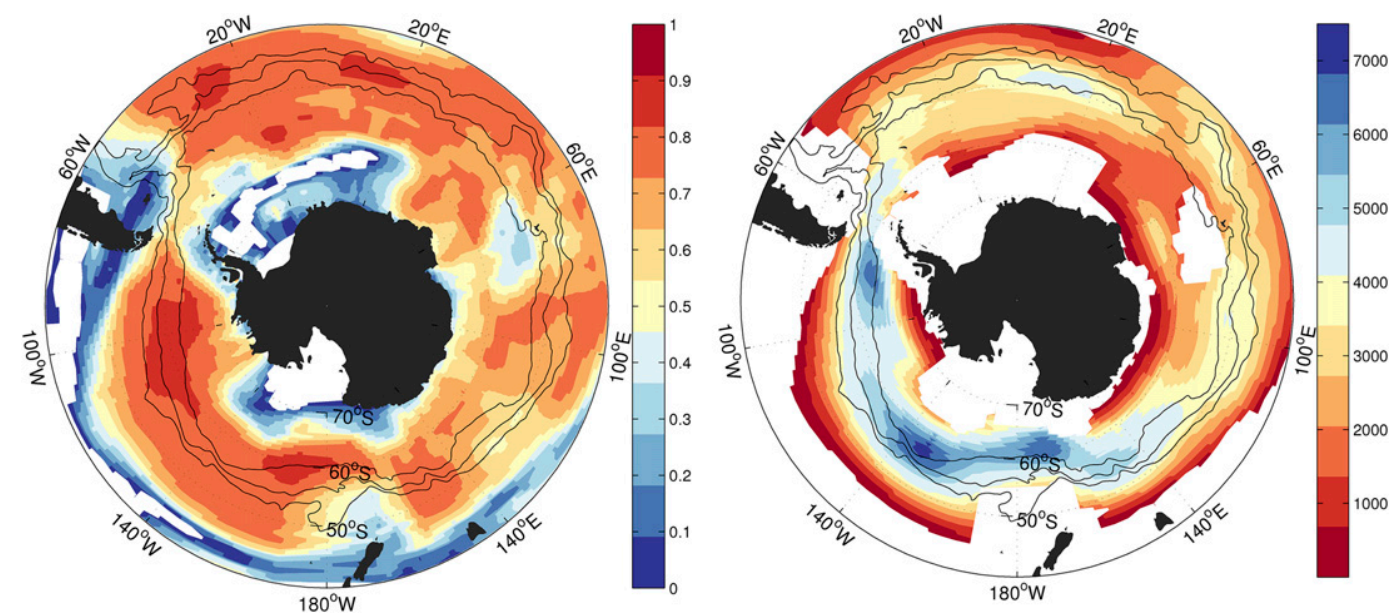

FIG. 4. (left) Values of $R^{2}$ for linear regressions between $M^{2}$ and $N^{2}$ from WOA13 six-decadal climatological temperature and salinity fields. Density is calculated using JMD95. Three ACC fronts are shown in solid black lines (SAF-N, SAF, and PF). Values are only plotted where the linear relationship is statistically significant at the $95 \%$ level or above (i.e., $f$ test $p<0.05$ ). (right) Vertical scale depth $H(\mathrm{~m})$ from WOA13 climatology. Values are only displayed where $R^{2}>0.5$ and $p<0.05$ for linear regressions between $M^{2}$ and $N^{2}$ at each latitude and longitude. The calculation is carried out in a moving window that is $20^{\circ}$ wide in longitude and $10^{\circ}$ wide in latitude.

for the Indian basin. Using Eq. (8) and the known value $f_{0}$, we can estimate the vertical scale $H$ for each of the three basins by linear regression. In the Atlantic sector $\left(20^{\circ} \mathrm{W}-0^{\circ}\right) H \approx 3000 \mathrm{~m}$, in the Indian sector $\left(90^{\circ}-110^{\circ} \mathrm{E}\right) H \approx 2600 \mathrm{~m}$, and in the Pacific sector $\left(130^{\circ}-110^{\circ} \mathrm{W}\right) H \approx 4900 \mathrm{~m}$. Since $H$ is the scale over which the slope of density surfaces changes significantly, $H$ can be larger than the actual depth of the ocean. Larger values of $H$ imply a slope that changes little with depth, relative to regions with smaller values of $H$. In the limit where the slope of density surfaces is uniform with depth, the scale $H$ approaches infinity.

We can estimate the degree to which $M^{2}$ and $N^{2}$ are linearly related by estimating the coefficient of determination (i.e., $R^{2}$ ) at each point on a latitudelongitude grid (Fig. 4). The variable $R^{2}$ can serve as a measure of the linear component in the relationship between $N^{2}$ and $M^{2}$ over broad patches of the Southern Ocean. The linearity between $N^{2}$ and $M^{2}$ is fairly strong (i.e., $R^{2}>0.7$ ) over most of the ACC, and is especially high in the Pacific (e.g., in the Bellingshausen basin, just upstream of Drake Passage). The coefficient $R^{2}$ becomes noticeably smaller (i.e., $R^{2}<$ $0.4)$ near large bathymetric features (e.g., Kerguelen Plateau, Campbell Plateau, and Falkland Plateau), and $R^{2}$ is especially small in the Ross and Weddell Seas, where sea ice melt and formation impose strong controls on the stratification. Near the Antarctic continent, the assumptions of quasigeostrophic theory are violated; the isopycnals are especially steep, and gyres dominate the dynamics. Since our scaling assumes a zonal mean structure, it should be most applicable across the ACC.

\section{c. Vertical scale of the slope gradient}

The Pacific sector of the Southern Ocean features especially weak vertical slope gradients. The Pacific basin is somewhat less topographically constrained than the other two basins, with relatively flat bottoms and no large plateaus. Subtropical stratification, which tends to be flatter and more uniform with depth, extends relatively far southward compared with the other two basins. It is interesting to note that the largest values of $H$ are found just south of (or slightly within) the Polar Front of the ACC.

\section{d. Sensitivity to averaging depth}

To test the sensitivity of the relationship between $M^{2}$ and $N^{2}$ to various parameters, we employ an idealized sector model as described in section 3 . Zonal mean density (i.e., potential density referenced to roughly $2000 \mathrm{~m}$ ) is depicted in Fig. 5a, wherein the averaging window is indicated with a dashed white line. The relationship between $M^{2}$ and $N^{2}$ depends on depth (Fig. 5b). If the averaging window is moved to the upper $300 \mathrm{~m}$ of the domain, then the vertical scale $H$ approaches zero (i.e., the slope changes extremely rapidly with depth thanks to the steep tilt). Below $1000 \mathrm{~m}$, the model ocean becomes nearly unstratified. As a result, both $M^{2}$ and $N^{2}$ tend toward zero. In this abyssal region, there is a 

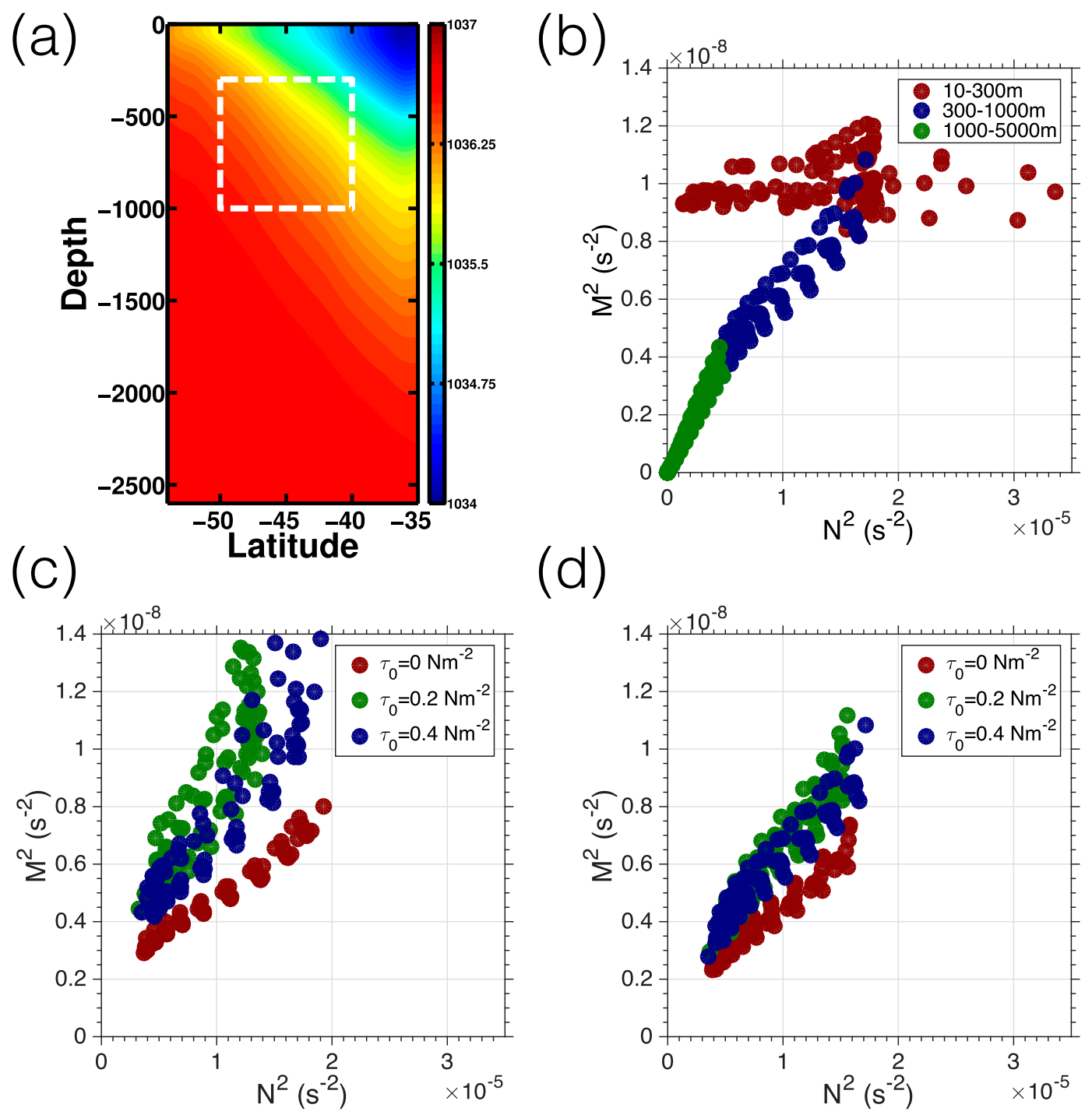

FIG. 5. (a) Zonal mean density $\left(\mathrm{kg} \mathrm{m}^{-3}\right)$ for the sector model. (b) Horizontal stratification vs vertical stratification for three different choices of vertical averaging scale. Values of $M^{2}$ vs $N^{2}$ for various values of maximum surface wind stress for a sector models with (c) $1^{\circ}$ horizontal resolution and (d) $1 / 6^{\circ}$ horizontal resolution. The white dashed line in (a) indicates the vertical averaging domain for plots (c) and (d). Since we are interested in large-scale features in this analysis, the $1 / 6^{\circ}$ model results were interpolated onto a $2^{\circ}$ grid.

strong linear relationship between the horizontal and vertical gradient in buoyancy. The quasigeostrophic scaling used in this paper is most appropriate between roughly 300 and $1000 \mathrm{~m}$ in the sector model, as indicated by the white box in Fig. 5a. The box is chosen to intersect the steeply tilted density surfaces of the circumpolar current while avoiding both the mixed layer and the weakly stratified deep ocean. Note the rapid slope change at approximately $1000 \mathrm{~m}$, which divides the vertical domain into an upper, rotationdominated region and a lower, stratification-dominated region.

\section{e. Sensitivity to wind stress and eddy activity}

Finally, we examine the sensitivity of the relationship between the horizontal and vertical stratification to resolved/permitted eddy activity (i.e., horizontal resolution) and wind stress. In Fig. 5c, we plot $M^{2}$ versus $N^{2}$ for three different maximum values of the surface wind stress $\left(0.0,0.2\right.$, and $\left.0.4 \mathrm{~N} \mathrm{~m}^{-2}\right)$. In comparing the coarseresolution case and the eddy-permitting case (i.e., Figs. $5 \mathrm{c}$ and $5 \mathrm{~d}$, respectively), we find that the scale $H$ is less sensitive to surface wind stress in the model with higher horizontal resolution (see Table 1 for estimates of 
TABLE 1 . Values of the depth scale $H(\mathrm{~m})$ obtained by the scaling $H=s /\left|\beta / f_{0}\right|$, where $s$ is the slope of the $M^{2} / N^{2}$ lines from sector model sensitivity experiments. Plots of $M^{2}$ vs $N^{2}$ are shown in Figs. $5 \mathrm{c}$ and $5 \mathrm{~d}$.

\begin{tabular}{lccc}
\hline \hline Wind stress max $\left(\mathrm{N} \mathrm{m}^{-2}\right)$ & 0.0 & 0.1 & 0.2 \\
\hline Horizontal resolution & & & \\
$2^{\circ}$ & 2100 & 3200 & 4800 \\
$1 / 6^{\circ}$ & 2100 & 2800 & 3300 \\
\hline
\end{tabular}

$H$ for the six different cases). The eddy-permitting model explicitly resolves large-scale eddies and is thereby better able to compensate for any wind-induced changes in isopycnal tilt. In the coarse-resolution model, eddies are parameterized following Gent and McWilliams (1990). In the presence of resolved/permitted mesoscale eddies, isopycnal slope is less sensitive to wind stress than when eddies are parameterized (Munday et al. 2013).

\section{f. Revisiting the assumptions}

In deriving Eq. (6), we made two assumptions in addition to those inherent to quasigeostrophic theory. First, we assumed that the ratio $\overline{\left|\partial_{y} \zeta_{g}\right|} / \beta \ll 1$, where $\zeta_{g}=$ $(\nabla \times \mathbf{v}) \cdot \mathbf{k}$ is the vertical component of relative vorticity. To check the validity of this assumption, we calculated $\zeta_{g}$ on the relatively fine (i.e., $16^{\circ}$ horizontal), threedimensional SOSE grid, and then we used a moving window that is $20^{\circ}$ wide in longitude, $10^{\circ}$ wide in latitude, and extends below $770 \mathrm{~m}$ and above $4825 \mathrm{~m}$ to calculate the large-scale average. The center of the moving window is placed on the centers of a $100 \times 100$ cell grid that are equally spaced in longitude and latitude, and each grid cell center is assigned the value of $\overline{\left|\partial_{y} \zeta_{g}\right|} / \beta$ estimated using the moving window centered there. Three fronts of the ACC are shown for reference (i.e., the SAF, SAF-N, and PF) (Sallée et al. 2008). The magnitude of the meridional gradient of relative vorticity is much smaller than $\beta$ nearly everywhere in the Southern Ocean, by one or two orders of magnitude in most locations (see Fig. 6a). This is consistent with the findings of Tulloch et al. (2011), wherein the authors used the Ocean Comprehensible Atlas (OCCA) to show that the surface relative vorticity is smaller than $\beta$ nearly everywhere in the global ocean by at least an order of magnitude (Forget 2010).

It should be noted that although the above scaling suggests that relative vorticity can be neglected in this analysis, relative vorticity can in principle have an impact on local potential vorticity gradients, leading to homogenization or even sharpening (Hughes 2005). The nonlinear component of vorticity advection can be important on smaller scales than those considered here (Hughes and Cuevas 2001).

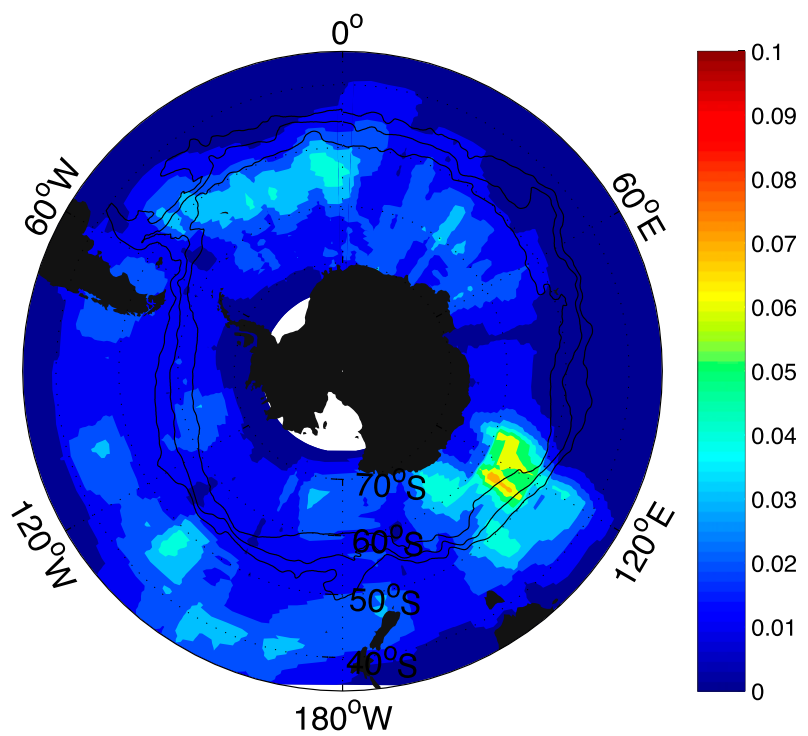

FIG. 6. Depth-averaged relative vorticity gradient in the Southern Ocean scaled by $\beta$. Zonal and meridional velocity fields from the Southern Ocean State Estimate (SOSE).

The second assumption that we made while deriving Eq. (6) was $\overline{\left|\partial_{y} q\right|} / \beta \ll 1$. This condition can hold in certain regions of the Southern Ocean. The observed density structure in the Southern Ocean as shown by Tulloch et al. (2011) using a climatology is replicated in Fig. 7, with a positive potential vorticity gradient in the upper water column and a negative potential vorticity gradient in the lower water column. The reversal of the sign of the meridional potential vorticity gradient with depth is a necessary condition for baroclinic instability, so we might expect to find such sign reversals in regions with steeply tilted isopycnals such as the Southern Ocean. With a suitable choice of averaging surface (e.g., box C in Fig. 7), the depth-averaged quasigeostrophic potential vorticity gradient vanishes, indicating a region where $r=1$ (i.e., the mean slope gradient is constrained by planetarygeostrophic parameters). The large-scale zonal mean meridional quasigeostrophic potential vorticity gradient is roughly of order $\beta$ over much of the Southern Ocean (Fig. 8). Suitable averaging volumes over which $\overline{\left|\partial_{y} q\right|} / \beta \ll 1$ can be found in the deep interior ocean (e.g., between $60^{\circ}$ and $50^{\circ} \mathrm{S}$ below roughly $2000 \mathrm{~m}$ ) and between the surface and thermocline at lower latitudes (e.g., between $40^{\circ}$ and $30^{\circ} \mathrm{S}$ from the surface down to about $2000 \mathrm{~m}$ ). However, even over many averaging volumes where $\overline{\left|\partial_{y} q\right|} / \beta \ll 1$ does not hold, the ratio $r$ remains order 1 across the contours of the ACC. The concept of the depth-averaged balance in Eq. (6) (i.e., the $r=1$ regime) provides a useful limiting case for understanding what sets isopycnal slope in rapidly rotating fluids with meridional potential vorticity gradient reversals. 


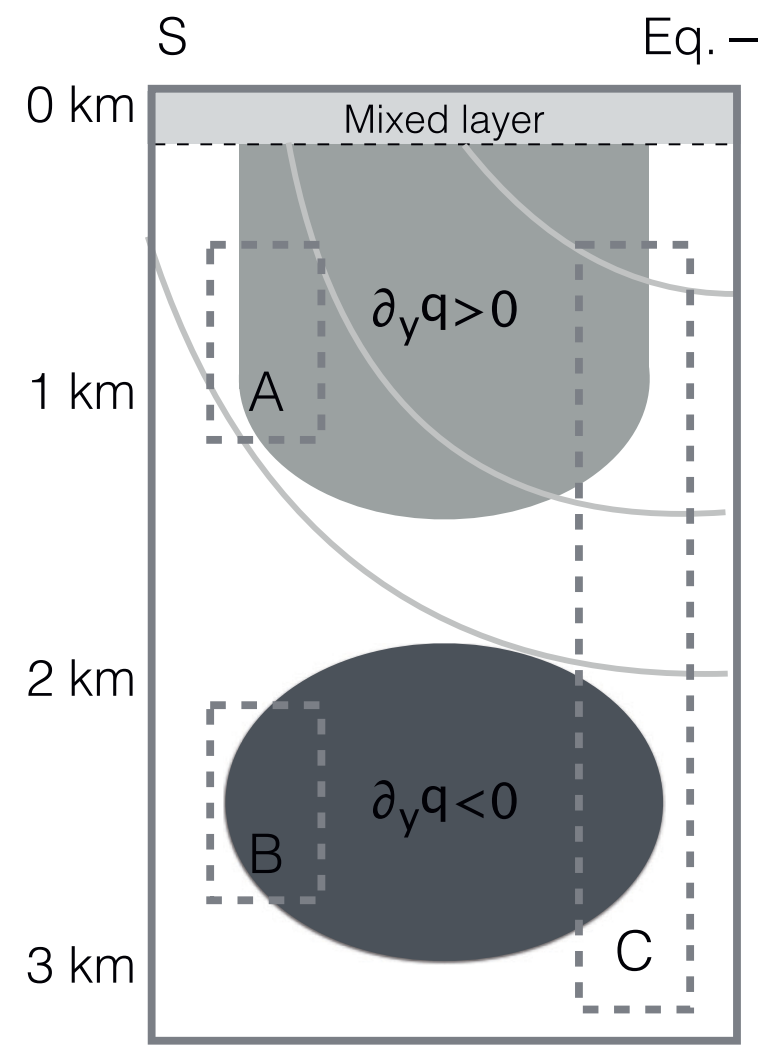

FIG. 7. Idealized schematic of zonal mean potential vorticity gradients (shading) and isopycnals (solid gray lines) in the Southern Ocean, adapted from Tulloch et al. (2011). The light shaded area indicates the region where the potential vorticity gradient is positive $\left(\partial_{y} q>0\right)$, and the dark shaded area indicates a region where the potential vorticity gradient is negative $\left(\partial_{y} q<0\right)$. The dashed boxes illustrate three different choices for vertical scales over which to average. The potential vorticity gradient is close to zero if the vertical averaging scale is chosen appropriately (i.e., for box $\mathrm{C}$ ). Averages taken over boxes $\mathrm{A}$ and $\mathrm{B}$ would have nonzero mean gradients.

In potential vorticity homogenization theory, eddies act to reduce potential vorticity gradients along isentropes, leading to meridional potential vorticity gradients that are much smaller than $\beta$. The $r=1$ condition is equivalent to the "strong constraint" that potential vorticity is completely homogenized. We have seen that the "strong" $r=1$ constraint does not hold in the Southern Ocean. However, a "weak" form of the constraint $r=O(1)$ does hold. In the Southern Ocean, $\beta$ itself is small, hence a potential vorticity gradient of order $\beta$ is still weakly consistent with the idea of potential vorticity homogenization.

\section{Discussion}

In this work, we have presented a planetarygeometric constraint that relates the depth-averaged

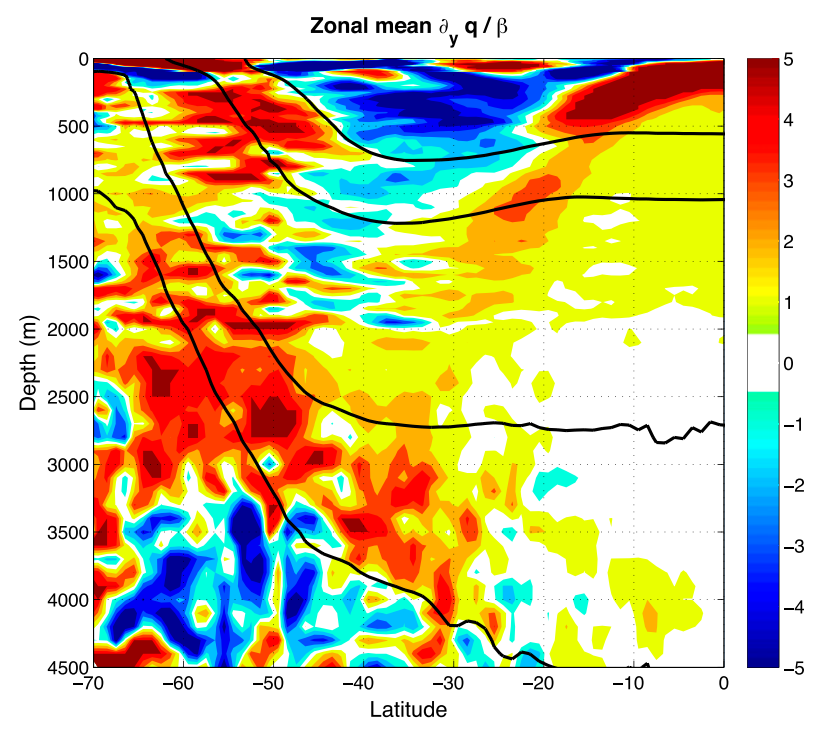

FIG. 8. Zonal mean quasigeostrophic potential vorticity from WOA13 six-decadal mean temperature and salinity. The values have been scaled by $\beta$. Zonal mean potential density contours are shown in black for $\sigma_{2}=36.0,36.5,37.0$, and $37.1 \mathrm{~kg} \mathrm{~m}^{-3}$. The reference buoyancy profile $\tilde{b}$ is defined by fitting a two-term exponential model [i.e. $\tilde{b}=a_{1} \exp \left(c_{1} z\right)+a_{2} \exp \left(c_{2} z\right)$ ] to zonal mean potential density $\sigma_{2}$ averaged over $60^{\circ}-55^{\circ} \mathrm{S}$.

vertical gradient of isopycnal slope to the ratio $\beta / f_{0}$. We have shown that this constraint holds, to first order, in the climatological Southern Ocean across the ACC, at least far from bathymetric obstructions. This result is broadly consistent with Jansen and Ferrari (2012), in which the authors discuss the sensitivity of stratification to planetary parameters (e.g., rotation rate). Our results are also consistent with detailed studies of the potential vorticity structure of the Southern Ocean [e.g., Marshall et al. 1993; Tulloch et al. 2011]. We used the relationship between horizontal and vertical stratification to derive a depth scale for the slope gradient. In this section, we discuss possible implications of the planetary-geometric constraint for large-scale ocean circulation and sensitivity.

\section{a. Thermal wind scaling}

We now translate the planetary-geometric constraint on isopycnal slope gradient into a constraint on a zonal velocity scale via the thermal wind relationship (i.e., $\left.\partial_{z} u=-f_{0}^{-1} \partial_{y} b\right)$. Assuming that thermal wind balance holds and using $s=-\partial_{y} b / \partial_{z} b$ to rewrite $\partial_{y} b$ in terms of $\partial_{z} b$, we take the vertical derivative of $\partial_{z} u=f_{0}^{-1} s \partial_{z} b$ to get

$$
\partial_{z z} u=f_{0}^{-1}\left(\partial_{z} s \partial_{z} b+s \partial_{z z} b\right) .
$$

The vertical average of Eq. (9) (denoted by an overbar) is 


$$
\begin{aligned}
\overline{\partial_{z z} u} & =f_{0}^{-1}\left(\overline{\partial_{z} s \partial_{z} b}+\overline{s \partial_{z z} b}\right) \\
& =f_{0}^{-1}\left[\overline{\partial_{z} s}\left(\overline{\partial_{z} b}\right)+\bar{s}\left(\overline{\partial_{z z} b}\right)+\overline{\partial_{z} s^{\prime} \partial_{z} b^{\prime}}+\overline{s^{\prime} \partial_{z z} b^{\prime}}\right]
\end{aligned}
$$

where we use the decomposition $\overline{a b}=\bar{a} \bar{b}+\overline{a^{\prime} b^{\prime}}$ to separate each vertical mean into a mean of products and a covariance term. Here, the primes denote departures from the vertical mean and not from the zonal mean as in previous sections. We can use the scaling $\overline{\partial_{z} s}=\beta f_{0}^{-1}$ to relate the slope gradient to $\beta / f_{0}$. If we take $H$ to be the depth scale for the slope gradient, we can also scale $\overline{\partial_{z} s}$ as $\bar{s} / H$, such that $\bar{s}=\beta f_{0}^{-1} H$. Assuming the covariance terms to be small (i.e., the mean buoyancy state dominates) and using the above scalings for the vertical slope gradient and the mean slope, we get $f_{0} \overline{\partial_{z z} u}=\beta f_{0}^{-1} N^{2}$, which scales as

$$
U_{\text {thermal }}=\frac{\beta N^{2} H^{2}}{f_{0}^{2}}=\beta L_{D}^{2}=-c,
$$

where $U_{\text {thermal }}$ is a zonal velocity scale for thermal wind balance, and we have used the baroclinic deformation radius $L_{D}=(N H) / f$ and the long Rossby wave speed $c=-\beta L_{D}^{2}$, where $c$ is the intrinsic phase speed for Rossby waves. This leads to

$$
r=\left|\frac{U_{\text {thermal }}}{c}\right|=1,
$$

where $r$, as in Eq. (5), is the depth-averaged criticality parameter. We can now use the result by Held and Larichev (1996) that the RMS eddy velocity $U_{\text {eddy }}$ is related to the mean thermal wind velocity through

$$
U_{\text {eddy }} \approx \frac{L}{L_{D}} U_{\text {thermal }},
$$

and noting that $L=L_{D}$ in the case where rotation and stratification are equally important, such as in the regime where $r=1$, to say [using Eq. (11)] that in a depthaverage large-scale sense $L=L_{D}=L_{\text {Rhines}}$, where $L_{\mathrm{Rhines}}=\sqrt{U_{\text {eddy }} / \beta}$ is the Rhines scale. This would suggest that in a depth-mean sense the length scales in the Southern Ocean are closely related to the Rhines scale.

\section{b. Eddy flux parameterization}

At present the horizontal resolution of most global ocean models is too coarse to represent the effects of mesoscale eddies, and hence their fluxes need to be parameterized. Here we focus on subgrid-scale fluxes of potential vorticity, since potential vorticity conservation provides a particularly strong constraint on largescale oceanic flow. Generally speaking, eddy fluxes of potential vorticity are associated with divergences and convergences of eddy fluxes of both buoyancy and momentum, that is,

$$
\overline{v^{\prime} q^{\prime}}=-\partial_{y}\left(\overline{u^{\prime} v^{\prime}}\right)+\partial_{z}\left(f_{0} N^{-2} \overline{v^{\prime} b^{\prime}}\right)
$$

where $u$ is the zonal velocity, $v$ is the meridional velocity, $q$ is the quasigeostrophic potential vorticity, and $b$ is the buoyancy. The overbars denote zonal means, and the primes denote departures from a zonal mean state. Therefore, $\overline{u^{\prime} v^{\prime}}$ represents eddy fluxes of momentum and $\overline{v^{\prime} b^{\prime}}$ represents eddy fluxes of buoyancy. A complete eddy flux parameterization would ideally contain representations of eddy fluxes of both momentum and buoyancy, although the relative importance of momentum and buoyancy fluxes is not obvious a priori.

Although there has been a flurry of recent activity regarding eddy parameterization (e.g., Viebahn and Eden 2010), many modern parameterization schemes are built on the foundation of Gent and McWilliams (1990, hereinafter GM90). In GM90, eddy buoyancy fluxes are parameterized through an eddy-induced transport velocity, which is represented as an additional velocity of the following form:

$$
\mathbf{u}^{*}=-\partial_{z}\left(\mathbf{K}_{\mathrm{GM}} \mathbf{s}_{b}\right),
$$

where $\mathbf{s}_{b}=-\nabla_{h} b / \partial_{z} b$ is the slope of buoyancy surfaces, $b$ is the locally referenced buoyancy, $\nabla_{h}$ is the horizontal gradient operator, and $\mathbf{K}_{\mathrm{GM}}$ is the diffusivity tensor. To date, eddy momentum fluxes are ignored in eddy parameterizations. The original GM90 formulation is given in terms of locally referenced potential density, but we use buoyancy here for consistency with the rest of this paper.

The GM90 scheme is built using an $f$-plane approximation (i.e., GM90 neglects the variation of the Coriolis parameter $f$ with latitude). On an $f$ plane, the isopycnal potential vorticity is clearly linked to the thickness of isopycnal surfaces. To be more specific, the QGPV gradient (neglecting relative vorticity as we have done throughout this paper) simplifies to $\partial_{y} q=-f_{0} \partial_{z} s_{b}$, where $s_{b}=-\partial_{\mathrm{y}} b / \partial_{\mathrm{z}} b$. In the absence of any forcing (e.g., no wind stress, no buoyancy fluxes), GM90 will tend to flatten isopycnals until they are completely horizontal, instead of flattening them until the $r=1$ regime is reached. This is consistent with Eq. (1) in the $f$-plane limit (i.e., $\overline{\partial_{y} q}=-f_{0} \overline{\partial_{z} s_{b}}$ ). Under such conditions, if $\overline{\partial_{y} q}=0$, then the average slope change is also zero (i.e., $\overline{\partial_{z} s_{b}}=0$ ), and the slope is uniform with depth (or steepens and tilts by about the same amount over the vertical domain). This is a reasonable approximation on smaller scales where changes in $f$ are small, but it does not hold on large spatial scales. 
GM90 uses Eq. (15) to parameterize the eddy-induced transport velocity, but the correct choice of $K_{\mathrm{GM}}$ is not obvious. At the moment, most models use ad hoc representations of $K_{\mathrm{GM}}$, with the most commonly used approach being a constant value for $K_{\mathrm{GM}}$, although there are exceptions [e.g., the spatially varying diffusivity of Visbeck et al. (1997) and Ferreira et al. (2005)]. Nevertheless, none of these approaches are likely to satisfy the $r=1$ constraint on large spatial scales. To correctly represent the $r=1$ regime using the GM90 eddy parameterization scheme, it is necessary to use physically meaningful values for the GM diffusion coefficient $K_{\mathrm{GM}}$. Smith and Marshall (2009) showed that this GM eddy diffusion coefficient, in a quasigeostrophic framework, is related to the isopycnal diffusion coefficient for potential vorticity $K_{q}$ via

$$
\frac{\partial}{\partial z}\left(K_{\mathrm{GM}} s_{b}\right)=K_{q}\left(\frac{\partial s_{b}}{\partial z}-\frac{\beta}{f}\right)
$$

where $K_{q}=-\overline{v^{\prime} q^{\prime}} / \partial_{y} q$. The lhs of Eq. (16) is just the (negative of the) eddy-induced transport velocity [Abernathey et al. (2013) have shown that this expression holds in a numerical model of an idealized ACC]. In this formulation, when $r=1$, the right-hand side of Eq. (16) is zero in a large-scale averaged sense, and therefore $\partial_{z}\left(K_{\mathrm{GM}} s_{b}\right)=0$. This zero eddy-induced velocity could be achieved in a depth-averaged sense if the eddy-induced velocity exactly compensates in the upper (lower) part of the water column where the potential vorticity gradient is positive (negative) (see Fig. 3). This shows that it is the role of eddy buoyancy fluxes to push the system back toward a state of marginal criticality.

Once the isopycnal eddy diffusivity is known [which can be calculated using mean flow and eddy properties; e.g., Klocker and Abernathey (2014)] and appropriate boundary conditions are chosen, it should be possible to derive a physically meaningful GM diffusion coefficient that flattens isopycnals only to the $r=1$ limit (i.e., where the slope gradient is constrained by the planetarygeometric parameter $\beta / f_{0}$ ) instead of to the $r=0$ limit (i.e., where the slope changes uniformly with depth). This approach has not yet been implemented in any ocean model, but this scheme would very likely lead to a much better representation of the ACC in coarse-resolution global climate models by correcting the equilibrium isopycnal slope across the ACC.

\section{Conclusions}

If the meridional gradients of relative vorticity (i.e., $\left.\partial_{y} \zeta_{g}\right)$ and potential vorticity (i.e., $\partial_{y} q$ ) are small relative to $\beta$, then quasigeostrophic theory predicts that the isopycnal slope $s$ is related to latitude $\phi_{0}$ and planetary radius $a$ by $d s / d z=\beta / f_{0}=\cot \left(\phi_{0}\right) / a$, or equivalently $r \equiv\left|\partial_{z} s /\left(\beta / f_{0}\right)\right|=1$, where $r$ is the depthaveraged criticality parameter. For large-scale climatological observations, we find that the strict $r=1$ condition holds over specific averaging volumes that include regions of both positive and negative meridional gradients of potential vorticity (i.e., $\partial_{y} q$ ) in roughly equal measures. A weaker $r=O(1)$ condition for depth-averaged values is generally satisfied along much of the Antarctic Circumpolar Current and throughout the wider Southern Ocean, particularly away from large bathymetric features. In these regions of the Southern Ocean, the large-scale average rate of change of slope with depth is constrained by $\beta / f_{0}$, a ratio of purely geometric parameters.

It is important to note that this scaling does not set isopycnal slope, but only its average vertical derivative. A change in forcing (e.g., an addition of buoyancy at high latitudes paired with a loss of buoyancy at low latitudes) may change the slope of isopycnal surfaces across the domain (e.g., box C in Fig. 7), but eddy activity will tend to restore the potential vorticity structure such that $r=O$ (1). This adjustment can in principle involve isopycnal steepening is some parts of the domain and flattening in others, which can change the baroclinic structure of the current; as long as the large-scale average slope gradient is of order $\beta / f_{0}$, the $r=O(1)$ constraint is satisfied.

The concept of the depth-averaged balance in Eq. (6) (i.e., the $r=1$ regime) provides a useful limiting case for understanding what sets isopycnal slope in rapidly rotating fluids with meridional potential vorticity gradient reversals. Although the Southern Ocean is an interesting test case for this concept, the $r=1$ balance may be useful for understanding changes in isopycnal slope in other systems with meridional potential vorticity gradient reversals. Possible applications may be found in paleoceanography and exoplanetary oceanography, which feature a wide range of surface buoyancy and wind forcing profiles. A more thorough exploration of the consequences of the $r=O(1)$ regime would make for a welcome addition to this discussion.

Acknowledgments. The authors thank Antoine Venaille, Andrew Meijers, Laure Zanna, Ryan Abernathey, Emma Boland, Trevor McDougall, and David Marshall for discussions and comments that greatly improved the quality of this paper. We also thank Jean-Baptiste Sallée for providing mean positions of the fronts of the Antarctic Circumpolar Current. DJ was supported by the Natural Environment Research Council (NERC Grant $\mathrm{NE} / \mathrm{J} 007757 / 1)$. This study is part of the British Antarctic 
Survey Polar Science for Planet Earth Programme. TI was supported by the National Science Foundation (1142009). AK was supported by an Australian Research Council Discovery Early Career Researcher Award (DE140100076). We gratefully acknowledge the thoughtful comments of two anonymous reviewers.

\section{APPENDIX A}

\section{Alternate Form of the Constraint}

If instead of using quasigeostrophic potential vorticity we use the planetary-geostrophic form:

$$
Q=f \frac{\partial b}{\partial z}=-\frac{f g}{\rho_{0}} \frac{\partial \rho}{\partial z}
$$

where $b=-g \rho_{0}^{-1}\left(\rho-\rho_{0}\right)$ [Vallis (2006), Eq. (5.52)], we can derive an alternate form of the constraint. Taking the meridional gradient, we have

$$
\partial_{y} Q=f g\left(\frac{\partial_{y z} \rho}{\rho_{0}}\right)+\beta g\left(\frac{\partial_{z} \rho}{\rho_{0}}\right) .
$$

Integrating from the bottom $B$ to the top $T$ of the interior ocean domain, we get

$$
\int_{B}^{T} \partial_{y} Q d z=f g \int_{B}^{T} \frac{\partial_{y z} \rho}{\rho_{0}} d z+\beta g \int_{B}^{T} \frac{\partial_{z} \rho}{\rho_{0}} d z .
$$

If we let $\Delta \rho \equiv \rho(T)-\rho(B)$, then we have

$$
\int_{B}^{T} \partial_{y} Q d z=\frac{g}{\rho_{0}}\left[f \partial_{y}(\Delta \rho)+\beta \Delta \rho\right] .
$$

Finally, if we take the depth-averaged meridional gradient of potential vorticity to be small (which is consistent with planetary-geostrophic scaling in the Southern Ocean), then the constraint on the density field can be written as

$$
\frac{\partial_{y}(\Delta \rho)}{\Delta \rho} \approx \frac{\beta}{f} .
$$

Equation (A5) is a constraint on the large-scale change in isopycnal slope between the top and bottom of the interior ocean domain.

\section{APPENDIX B}

\section{Vertical Velocity Scales}

The evolution of quasigeostrophic potential vorticity is described by the prognostic equation

$$
\frac{\partial q}{\partial t}+u \frac{\partial q}{\partial x}+v \frac{\partial q}{\partial y}+w \frac{\partial q}{\partial z}=F
$$

where $F$ represents any nonconservative processes. Let $q$ be a steady-state zonal mean quantity (such that $\partial_{t} q=$ 0 and $\left.\partial_{x} q=0\right)$ and use $\overline{\partial_{y} q}=\beta(1-r)$ to relate the meridional potential vorticity gradient to $r$ and $\beta$ :

$$
v \beta(1-r)+w \frac{\partial q}{\partial z}=F .
$$

Using the quasigeostrophic definition of potential vorticity:

$$
v \beta(1-r)+w \partial_{z z}\left(f_{0} N^{-2} b^{\prime}\right)=F .
$$

Solving for $w$, we get

$$
w=\frac{F+\beta v(r-1)}{f_{0} \partial_{z z}\left(N^{-2} b^{\prime}\right)} .
$$

That is, the vertical and horizontal velocities are connected by the planetary-geometric parameters (i.e., $r, \beta$, and $f_{0}$ ) and the vertical density structure (i.e., $N^{2}$ and $b$ ). Simulations find that upwelling is focused in intermediate layers where significant potential vorticity gradients exist (i.e., where $F=0$ and $r \neq 1$ ) (Hallberg and Gnanadesikan 2006; Farneti et al. 2010), which is consistent with the above formulation. In the simple conservative case (i.e., $F=0$ ), if $v$ has an equivalent barotropic structure, then $w$ is constrained by this equivalent barotropic structure under simple stratification conditions. Furthermore, if $r=1$, then $w=0$. That is, weak vertical velocities are consistent with small horizontal potential vorticity gradients in a bulk-average sense. Care should be taken when interpreting Eq. (B4), since $r$ is strictly a large-scale bulk metric that should only be applied to the interior ocean.

\section{REFERENCES}

Abernathey, R., J. Marshall, and D. Ferreira, 2011: The dependence of Southern Ocean meridional overturning on wind stress. J. Phys. Oceanogr., 41, 2261-2278, doi:10.1175/ JPO-D-11-023.1.

, D. Ferreira, and A. Klocker, 2013: Diagnostics of isopycnal mixing in a circumpolar channel. Ocean Modell., 72, 1-16, doi:10.1016/j.ocemod.2013.07.004.

Bretherton, F., 1966: Baroclinic instability and the short wavelength cutoff in terms of potential vorticity. Quart. J. Roy. Meteor. Soc., 92, 335-345, doi:10.1002/qj.49709239303.

Delworth, T., and Coauthors, 2012: Simulated climate and climate change in the GFDL CM2.5 high-resolution coupled climate model. J. Climate, 25, 2755-2781, doi:10.1175/ JCLI-D-11-00316.1. 
Farneti, R., T. L. Delworth, A. J. Rosati, S. M. Griffies, and F. Zeng, 2010: The role of mesoscale eddies in the rectification of the Southern Ocean response to climate change. J. Phys. Oceanogr., 40, 1539-1557, doi:10.1175/2010JPO4353.1.

Ferreira, D., J. Marshall, and P. Heimbach, 2005: Estimating eddy stresses by fitting dynamics to observations using a residual-mean ocean circulation model and its adjoint. J. Phys. Oceanogr., 35, 1891-1910, doi:10.1175/JPO2785.1.

Forget, G., 2010: Mapping ocean observations in a dynamical framework: A 2004-06 ocean atlas. J. Phys. Oceanogr., 40, 1201-1221, doi:10.1175/2009JPO4043.1.

Gent, P., and J. McWilliams, 1990: Isopycnal mixing in ocean circulation models. J. Phys. Oceanogr., 20, 150-155, doi:10.1175/ 1520-0485(1990)020<0150:IMIOCM > 2.0.CO;2.

Hallberg, R., and A. Gnanadesikan, 2006: The role of eddies in determining the structure and response of the wind-driven Southern Hemisphere overturning: Results from the modeling eddies in the Southern Ocean (MESO) project. J. Phys. Oceanogr., 36, 2232-2252, doi:10.1175/JPO2980.1.

Held, I. M., and V. D. Larichev, 1996: A scaling theory for horizontally homogeneous, baroclinically unstable flow on a beta plane. J. Atmos. Sci., 53, 946-952, doi:10.1175/ 1520-0469(1996)053<0946:ASTFHH > 2.0.CO;2.

Hogg, A., and D. Munday, 2014: Does the sensitivity of Southern Ocean circulation depend upon bathymetric details? Philos. Trans. Roy. Soc. London, 372A, 2019, doi:10.1098/ rsta.2013.0050.

Hughes, C., 2005: Nonlinear vorticity balance of the Antarctic Circumpolar Current. J. Geophys. Res., 110, C11008, doi:10.1029/2004JC002753.

_ realistic oceans are inviscid: A link between form stress and bottom pressure torques. J. Phys. Oceanogr., 31, 2871-2885, doi:10.1175/1520-0485(2001)031<2871:WWBCIR >2.0.CO;2.

Jackett, D., and T. Mcdougall, 1995: Minimal adjustment of hydrographic profiles to achieve static stability. J. Atmos. Oceanic Technol., 12, 381-389, doi:10.1175/1520-0426(1995)012<0381: MAOHPT $>2.0 . \mathrm{CO} ; 2$.

Jansen, M., and R. Ferrari, 2012: Macroturbulent equilibration in a thermally forced primitive equation system. J. Atmos. Sci., 69 , 695-713, doi:10.1175/JAS-D-11-041.1.

Karsten, R., and J. Marshall, 2002: Constructing the residual circulation of the ACC from observations. J. Phys. Oceanogr., 32, 3315-3327, doi:10.1175/1520-0485(2002)032<3315: CTRCOT $>2.0 . \mathrm{CO} ; 2$.

Klocker, A., and R. Abernathey, 2014: Global patterns of mesoscale eddy properties and diffusivities. J. Phys. Oceanogr., 44, 1030-1046, doi:10.1175/JPO-D-13-0159.1.

Locarnini, R. A., A. V. Mishonov, J. I. Antonov, T. P. Boyer, H. E. Garcia, O. K. Baranova, M. M. Zweng, and D. R. Johnson, 2010: Temperature. Vol. 1, World Ocean Atlas 2009, NOAA Atlas NESDIS 68, $184 \mathrm{pp}$.

Lovenduski, N., and T. Ito, 2009: The future evolution of the Southern Ocean $\mathrm{CO}_{2}$ sink. J. Mar. Res., 67, 597-617, doi:10.1357/002224009791218832.

Marshall, J., and T. Radko, 2003: Residual-mean solutions for the Antarctic Circumpolar Current and its associated overturning circulation. J. Phys. Oceanogr., 33, 2341-2354, doi:10.1175/ 1520-0485(2003)033<2341:RSFTAC >2.0.CO;2. and K. Speer, 2012: Closure of the meridional overturning circulation through Southern Ocean upwelling. Nat. Geosci., 5, 171-180, doi:10.1038/ngeo1391.

— D. Olbers, H. Ross, and D. Wolf-Gladrow, 1993: Potential vorticity constraints on the dynamics and hydrography of the Southern Ocean. J. Phys. Oceanogr., 23, 465-487, doi:10.1175/ 1520-0485(1993)023<0465:PVCOTD > 2.0.CO;2.

—, A. Adcroft, C. Hill, and L. Perelman, 1997a: A finite-volume, incompressible Navier-Stokes model for studies of the ocean on parallel computers. J. Geophys. Res., 102, 5753-5766, doi:10.1029/96JC02775.

— C. Hill, L. Perelman, and A. Adcroft, 1997b: Hydrostatic, quasi-hydrostatic, and nonhydrostatic ocean modeling. J. Geophys. Res., 102, 5733-5752, doi:10.1029/96JC02776.

Mazloff, M., P. Heimbach, and C. Wunsch, 2010: An eddypermitting Southern Ocean state estimate. J. Phys. Oceanogr., 40, 880-899, doi:10.1175/2009JPO4236.1.

Munday, D. R., H. L. Johnson, and D. P. Marshall, 2013: Eddy saturation of equilibrated circumpolar currents. J. Phys. Oceanogr., 43, 507-532, doi:10.1175/JPO-D-12-095.1.

$\longrightarrow, \ldots$, and — 2014: Impacts and effects of mesoscale ocean eddies on ocean carbon storage and atmospheric $\mathrm{pCO}_{2}$. Global Biogeochem. Cycles, 28, 877-896, doi:10.1002/ 2014GB004836.

Munk, W., and E. Palmén, 1951: Note on the dynamics of the Antarctic Circumpolar Current. Tellus, 3A, 53-55, doi:10.1111/ j.2153-3490.1951.tb00776.x.

Russell, J., K. Dixon, A. Gnanadesikan, R. Stouffer, and J. Toggweiler, 2006: The Southern Hemisphere westerlies in a warming world: Propping open the door to the deep ocean. J. Climate, 19, 6382-6390, doi:10.1175/ JCLI3984.1.

Sallée, J., K. Speer, and R. Morrow, 2008: Response of the Antarctic Circumpolar Current to atmospheric variability. J. Climate, 21, 3020-3039, doi:10.1175/2007JCLI1702.1.

Smith, K., and J. Marshall, 2009: Evidence for enhanced eddy mixing at middepth in the Southern Ocean. J. Phys. Oceanogr., 39, 50-69, doi:10.1175/2008JPO3880.1.

Stone, P., 1978: Baroclinic adjustment. J. Atmos. Sci., 35, 561-571, doi:10.1175/1520-0469(1978)035<0561:BA > 2.0.CO;2.

Takahashi, T., and Coauthors, 2009: Climatological mean and decadal change in surface ocean $\mathrm{pCO}_{2}$, and net sea-air $\mathrm{CO}_{2}$ flux over the global oceans. Deep-Sea Res. II, 56, 554-577, doi:10.1016/j.dsr2.2008.12.009.

Tulloch, R., J. Marshall, C. Hill, and K. S. Smith, 2011: Scales, growth rates, and spectral fluxes of baroclinic instability in the ocean. J. Phys. Oceanogr., 41, 1057-1076, doi:10.1175/2011JPO4404.1.

Vallis, G. K., 2006: Atmospheric and Oceanic Fluid Dynamics: Fundamentals and Large-Scale Circulation. Cambridge University Press, 769 pp.

Viebahn, J., and C. Eden, 2010: Towards the impact of eddies on the response of the Southern Ocean to climate change. Ocean Modell., 34, 150-165, doi:10.1016/ j.ocemod.2010.05.005.

Visbeck, M., J. Marshall, and T. Haine, 1997: Specification of eddy transfer coefficients in coarse-resolution ocean circulation models. J. Phys. Oceanogr., 27, 381-402, doi:10.1175/ 1520-0485(1997)027<0381:SOETCI >2.0.CO;2.

Zweng, M. M., and Coauthors, 2013: Salinity. Vol. 2, World Ocean Atlas 2013, NOAA Atlas NESDIS 74, 39 pp. 